\title{
QUANTIZATION OF $A_{0}(K)$-SPACES
}

\author{
ANINDYA GHATAK AND ANIL KUMAR KARN
}

Abstract. Let $\left(V,\left\{\|\cdot\|_{n}\right\},\left\{M_{n}(V)^{+}\right\}\right)$be a $C^{*}$-ordered operator space and $Q_{n}(V)$ be the quasistate space of $M_{n}(V)$. We show that every $\mathrm{C}^{*}$-ordered operator space $V$ is complete isometrically, completely isomorphic to $\left\{A_{0}\left(Q_{n}(V), M_{n}\left(V^{*}\right)\right)\right\}$. Motivated by this result we study matricial convexity property. We introduce a notion of an $L^{1}$-matrix convex set $\left\{K_{n}\right\}$ in a $*$ locally convex space $X$. We show that every quantized function space $\left\{A_{0}\left(K_{n}, M_{n}(X)\right)\right\}$ is a $C^{*}$-ordered operator space. Further, we generalize the notion of regular embedding of a compact convex set to $L^{1}$-regular embedding of an $L^{1}$-matrix convex set. We show that if a $L^{1}$-matricial convex set is $L^{1}$-regular embed and $L^{1}$-matricial cap, then $A_{0}\left(K_{n}, M_{n}(V)\right)$ is an abstract operator system.

Mathematics subject classification (2010): 46B40, 46L07, 47L25.

Keywords and phrases: Operator space, operator system, $\mathrm{C}^{*}$-ordered operator space, matrix convex set, $L^{1}$-Matrix convex set.

\section{REFERENCES}

[1] E. M. Alfsen, Compact Convex Sets and Bounded Integrals, 57, Springer Verlag, Berlin-HeidelbergNew York, (1971).

[2] L. Asimov, Well-capped convex cones, Pacific J. Math, 26, (1968), 421-431.

[3] M. D. Chol, E. G. EfFros, Injectivity and Operator Spaces, J. Func. Anal., 24 (1977), 156-209.

[4] E. G. EfFors, Z. J. RuAn, On the Abstract Characterization of Operator Spaces, Proc. Amer. Math. Soc., 119, (1993), 579-584.

[5] A. Ghatak, A. Karn, CM-ideals and $L^{1}$-matricial split faces, Acta Sci. Math. (Szeged) 85 (2019), 659-679.

[6] G. J. O. Jameson, Order Linear Spaces, Springer-Verlag, Lecture Notes No. 141, Berlin, (1970).

[7] A. YA. Helems KiI, Quantum Functional Analysis: Non-Coordinate Approach, University Lecture Series, 56. American Mathematical Society, Providence, RI, (2010).

[8] R. V. Kadison, A Representation Theory for Commutative Topological Algebras, Mem. Amer. Math. Soc. 7, (1951).

[9] A. K. KARn, Order Embedding of Matrix Ordered Spaces, Bull. Aust. Math. Soc., 84, (2011) 10-18.

[10] A. K. KARN, A p-Theory of Order Normed Spaces, Positivity, 14, (2010), 441-458.

[11] K. F. NG, The Duality of Partially Ordered Banach Spaces, Proc. Lond. Math. Soc., 3, (1969), 269288.

[12] Z. J. RuAn, Subspaces of C* -Algebras, J. Func. Anal., 29, (1998), 217-230.

[13] C. Webster, S. Winkler, Krein Milman Theorem for Operator Convexity, Trans. Amer. Math. Soc., 351, (1999), 307-322.

[14] W. WERnER, Multipliers on matrix ordered operator spaces and some $K$-groups, J. Funct. Anal. 206 (2004), 356-378.

[15] W. Werner, Subspaces of $L(H)$ that are *-invariant, J. Funct. Anal. 193 (2002), 207-223.

[16] Y. C. Wong, F. G. Kung, Partially ordered topologically vector spaces, Oxford Mathematical Monographs. Clarendon Press, Oxford, (1973). 